\title{
A non-overlapping Schwarz domain decomposition method with high-order finite elements for flow acoustics
}

\author{
Alice Lieu $^{\mathrm{a}}$, Philippe Marchner ${ }^{\mathrm{b}, \mathrm{d}, \mathrm{e}, *}$, Gwénaël Gabard $^{\mathrm{c}}$, Hadrien Bériot ${ }^{\mathrm{b}}$, Xavier Antoine $^{\mathrm{d}}$, \\ Christophe Geuzaine \\ ${ }^{\text {a } I n s t i t u t e ~ o f ~ S o u n d ~ a n d ~ V i b r a t i o n ~ R e s e a r c h, ~ U n i v e r s i t y ~ o f ~ S o u t h a m p t o n, ~ U K ~}$ \\ ${ }^{\mathrm{b}}$ Siemens Industry Software SAS, 150 Avenue de la République, 92320 Châtillon, France \\ ${ }^{\mathrm{c}}$ LAUM, Le Mans Université, 72000 Le Mans Cedex 9, France \\ ${ }^{\mathrm{d}}$ Institut Elie Cartan de Lorraine, UMR CNRS 7502, Université de Lorraine, Inria Nancy-Grand Est, SPHINX Team, 54506 \\ Vandoeuvre-lès-Nancy Cedex, France

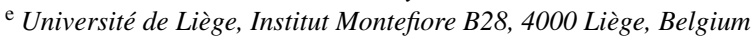

Received 19 November 2019; received in revised form 17 June 2020; accepted 17 June 2020

Available online $\mathrm{xxxx}$

\begin{abstract}
A non-overlapping domain decomposition method is proposed to solve large-scale finite element models for the propagation of sound with a background mean flow. An additive Schwarz algorithm is used to split the computational domain into a collection of sub-domains, and an iterative solution procedure is formulated in terms of unknowns defined on the interfaces between sub-domains. This approach allows to solve large-scale problems in parallel with only a fraction of the memory requirements compared to the standard approach which is to use a direct solver for the complete problem. While domain decomposition techniques have been used extensively for Helmholtz problems, this is the first application to aero-acoustics. The optimized Schwarz formulation is extended to the linearized potential theory for sound waves propagating in a potential base flow. A high-order finite element method is used to solve the governing equations in each sub-domain, and well-designed interface conditions based on local approximations of the Dirichlet-to-Neumann map are used to accelerate the convergence of the iterative procedure. The method is assessed on an academic test case and its benefit demonstrated on a realistic turbofan engine intake configuration.
\end{abstract}

(C) 2020 Elsevier B.V. All rights reserved.

Keywords: Domain decomposition; Optimized Schwarz method; Flow acoustics; Transmission conditions; High-order finite element; Turbofan intake

\section{Introduction}

Computational models predicting the propagation and radiation of noise from aeroengines remain a crucial tool to support the acoustic design of various components of turbofan engines, an obvious example being the optimization of acoustic liners on the nacelle of the engine [1]. Arguably the most widespread scheme for this purpose is the

\footnotetext{
* Corresponding author at: Siemens Industry Software SAS, 150 Avenue de la République, 92320 Châtillon, France.

E-mail addresses: philippe.marchner@siemens.com (P. Marchner), gwenael.gabard@univ-lemans.fr (G. Gabard), hadrien.beriot@siemens.com (H. Bériot), xavier.antoine@univ-lorraine.fr (X. Antoine), cgeuzaine@uliege.be (C. Geuzaine).
} 
linearized potential equation solved in the frequency domain with standard finite elements. This approach forms the basis for commercial codes routinely used in industry. One of the main limitations of this approach is the computational resources and time. The upper frequency limit that can be considered is dictated by the memory available which represents the main bottleneck when solving large-scale finite element models in the frequency domain.

Using a domain decomposition method allows to reduce significantly the memory requirements by splitting the computational domain into sub-domains. A direct solver is used for each sub-domain, but the global solution is obtained through an iterative procedure, thus reducing the amount of memory needed. This type of method has been used extensively in classical acoustics [2-5], or electromagnetism [6,5,7,8], but this paper presents the application of a non-overlapping domain decomposition approach to sound propagation with a background mean flow. In this work the optimized Schwarz method is formulated for the linearized potential equation. The method is equivalent to the FETI-2LM approach (Finite Element Tearing and Interconnecting - 2 Lagrange Multipliers) which was proposed earlier for the Helmholtz equation [9] as an extension to the initial work of Després [10]. In FETI-2LM, the Lagrange multipliers play the role of the interface unknowns defined in optimized Schwarz methods. The latter further generalize the choice of the interface conditions by defining them at the continuous level, while FETI has historically been designed at the algebraic level. In what follows, we will therefore use the formalism of optimized Schwarz methods with general transmission conditions such as presented in [5]. In each sub-domain the governing equation is solved using a high-order finite element method which allows to further reduce the computational costs. The method is assessed on the scattering of a rigid cylinder in a potential flow and then applied to a turbofan engine intake.

The paper is organized as follows. The linearized potential theory is described in the next section, followed by a description of the domain decomposition framework where the equivalence between FETI and Schwarz-type methods is clarified. The high-order finite element method is then presented in Section 4, and examples of results are given in Section 5 to illustrate the benefit of the domain decomposition method compared to the standard approach. The performance of different interface conditions is examined in order to improve the efficiency of the iterative solver. Finally, three-dimensional parallel computations with a strong background mean flow are shown in Section 6.

\section{Sound propagation model}

We model the propagation of sound waves in a steady, potential base flow using the linearized potential equation:

$$
\rho_{0} \frac{\mathrm{D}_{0}}{\mathrm{D} t}\left(\frac{1}{c_{0}^{2}} \frac{\mathrm{D}_{0} \phi}{\mathrm{D} t}\right)-\nabla \cdot\left(\rho_{0} \nabla \phi\right)=0,
$$

where $\mathrm{D}_{0} / \mathrm{D} t=\partial / \partial t+\mathbf{u}_{0} \cdot \nabla$ represents the material derivative in the mean flow $\mathbf{u}_{0}$. The mean sound speed and density are denoted by $c_{0}$ and $\rho_{0}$. With this propagation model the sound field is described by the scalar potential $\phi$ from which the acoustic velocity is derived $(\mathbf{u}=\nabla \phi)$. The acoustic pressure is also recovered using $p=-\rho_{0} \mathrm{D}_{0} \phi / \mathrm{D} t$. This model represents the convective effect of the flow on the sound field, but does not include the refraction effect that would be induced by a sheared mean flow. It is however well suited for applications such as the radiation of sound from turbofan intakes. The wave equation (1) is solved in the frequency domain by assuming a $\mathrm{e}^{\mathrm{i} \mathrm{i} \omega t}$ time dependence.

For the purpose of predicting noise radiation from aeroengines various boundary conditions should be considered. The condition for hard-wall surfaces is a vanishing normal acoustic velocity: $\mathbf{u} \cdot \mathbf{n}=\partial \phi / \partial n=0$ where $\mathbf{n}$ is the outward-pointing normal. To describe an acoustically treated surface the Ingard-Myers boundary condition is used [11]. It takes into account an infinitely thin boundary layer above the liner. To represent the scattered field to infinity, Perfectly Matched Layers (PML) are implemented around the boundary of the domain to absorb outgoing waves and remove spurious reflections $[12,13]$. The PML can also be used to generate a sound field entering the computational domain. This so-called 'active' PML is used to define the sources of sound radiating from the engine, typically from the fan, which are generally described in terms of acoustic duct modes.

The sound radiated to the far-field is computed using a Kirchhoff surface that allows to extrapolate the near-field solution to the far-field. The Kirchhoff formulation assumes a uniform mean flow outside of the control surface. 


\section{Non-overlapping domain decomposition}

We now present how the domain decomposition method can be applied to solve Eq. (1). The computational domain $\Omega$ is subdivided into non-overlapping sub-domains $\Omega_{i}$, with $i=1, \ldots, N_{\mathrm{s}}$, where $N_{\mathrm{s}}$ is the number of sub-domains. For brevity and clarity, the formulation will be presented for two domains $i$ and $j$, but the procedure extends to an arbitrary number of sub-domains.

The variational formulations associated with Eq. (1) written in sub-domains $\Omega_{i}$ and $\Omega_{j}$ read

$$
\begin{aligned}
& \int_{\Omega_{i}}\left[\rho_{0} \overline{\nabla \psi_{i}} \cdot \nabla \phi_{i}-\frac{\rho_{0}}{c_{0}^{2}} \frac{\overline{\mathrm{D}_{0} \psi_{i}}}{\mathrm{D} t} \frac{\mathrm{D}_{0} \phi_{i}}{\mathrm{D} t}\right] \mathrm{d} \Omega+\int_{\partial \Omega_{i}} \overline{\psi_{i}}\left[\frac{\rho_{0}}{c_{0}^{2}} \frac{\mathrm{D}_{0} \phi_{i}}{\mathrm{D} t}\left(\mathbf{u}_{0} \cdot \mathbf{n}_{i}\right)-\rho_{0} \frac{\partial \phi_{i}}{\partial n_{i}}\right] \mathrm{d} S=0, \\
& \int_{\Omega_{j}}\left[\rho_{0} \overline{\nabla \psi_{j}} \cdot \nabla \phi_{j}-\frac{\rho_{0}}{c_{0}^{2}} \frac{\overline{\mathrm{D}_{0} \psi_{j}}}{\mathrm{D} t} \frac{\mathrm{D}_{0} \phi_{j}}{\mathrm{D} t}\right] \mathrm{d} \Omega+\int_{\partial \Omega_{j}} \overline{\psi_{j}}\left[\frac{\rho_{0}}{c_{0}^{2}} \frac{\mathrm{D}_{0} \phi_{j}}{\mathrm{D} t}\left(\mathbf{u}_{0} \cdot \mathbf{n}_{j}\right)-\rho_{0} \frac{\partial \phi_{j}}{\partial n_{j}}\right] \mathrm{d} S=0,
\end{aligned}
$$

where $\psi_{i}$ and $\psi_{j}$ are the test-functions associated to the fields $\phi_{i}$ and $\phi_{j}$. The unit vectors $\mathbf{n}_{i}$ and $\mathbf{n}_{j}$ are the outward-pointing normals to the boundaries $\partial \Omega_{i}$ and $\partial \Omega_{j}$. The notation $\div$ represents the complex conjugate. The sub-domains $\Omega_{i}$ and $\Omega_{j}$ share a common boundary interface denoted $\Gamma_{i j}$. The terms involved on the interface $\Gamma_{i j}$ are written as follows

$$
\int_{\Gamma_{i j}}\left[\overline{\psi_{i}} \mathcal{B}_{i} \phi_{i}+\overline{\psi_{j}} \mathcal{B}_{j} \phi_{j}\right] \mathrm{d} \Gamma
$$

where we have identified the boundary operator involved on $\Gamma_{i j}$ :

$$
\mathcal{B}_{i} \phi_{i}=\frac{\rho_{0}}{c_{0}^{2}} \frac{\mathrm{D}_{0} \phi_{i}}{\mathrm{D} t}\left(\mathbf{u}_{0} \cdot \mathbf{n}_{i}\right)-\rho_{0} \frac{\partial \phi_{i}}{\partial n_{i}}=\rho_{0} \mathrm{i} k_{0} M_{n_{i}} \phi_{i}+\rho_{0} M_{n_{i}} M_{\tau_{i}} \frac{\partial \phi_{i}}{\partial \tau_{i}}+\rho_{0}\left(M_{n_{i}}^{2}-1\right) \frac{\partial \phi_{i}}{\partial n_{i}},
$$

and similarly for $\mathcal{B}_{j} \phi_{j}$. We have introduced the local Mach number $M=\left\|\mathbf{u}_{0}\right\| / c_{0}$, the free-field wavenumber $k_{0}=\omega / c_{0}$ and the unit tangential vectors $\boldsymbol{\tau}_{i}$ and $\boldsymbol{\tau}_{j}$ along $\partial \Omega_{i}$ and $\partial \Omega_{j}$. The Mach numbers relative to the normal $\mathbf{n}_{i}$ and tangential $\boldsymbol{\tau}_{i}$ components of the mean flow are denoted by $M_{n_{i}}$ and $M_{\tau_{i}}$. Note that for the Helmholtz equation (i.e. for a uniform quiescent medium) the boundary operators reduce to the usual normal derivatives: $\mathcal{B}_{i} \phi_{i}=-\rho_{0} \partial \phi_{i} / \partial n_{i}$ and $\mathcal{B}_{j} \phi_{j}=-\rho_{0} \partial \phi_{j} / \partial n_{j}$.

Between the two domains we would like to impose the continuity of the unknown field $\phi$ and its normal derivative $\partial \phi / \partial n$. Applying these two conditions directly in the variational formulations above results in a model that is ill-conditioned, see [14, section 2.2.1] and [10,15]. Optimized Schwarz methods provide a well-defined formulation $[5,16]$.

\subsection{Sub-domains coupling - transmission operators}

To introduce a coupling between the two sub-domains, the last term in the boundary operator defined in Eq. (4) is rewritten as follows:

$$
\begin{gathered}
\rho_{0}\left(M_{n_{i}}^{2}-1\right) \frac{\partial \phi_{i}}{\partial n_{i}}=\rho_{0}\left(M_{n_{i}}^{2}-1\right) \mathcal{S}_{i} \phi_{i}+\mathcal{L}_{j \rightarrow i} \phi_{j}, \\
\rho_{0}\left(M_{n_{j}}^{2}-1\right) \frac{\partial \phi_{j}}{\partial n_{j}}=\rho_{0}\left(M_{n_{j}}^{2}-1\right) \mathcal{S}_{j} \phi_{j}+\mathcal{L}_{i \rightarrow j} \phi_{i},
\end{gathered}
$$

where $\mathcal{L}_{i \rightarrow j}$ and $\mathcal{L}_{j \rightarrow i}$ are the transmission operators that couple the two solutions $\phi_{i}$ and $\phi_{j}$ on the interface $\Gamma_{i, j}$. The operators $\mathcal{S}_{i}$ and $\mathcal{S}_{j}$ replace the $\partial \phi / \partial n$ terms and act as non-reflecting operators for the domains $\Omega_{i}$ and $\Omega_{j}$. The optimal non-reflecting operators would be the Dirichlet-to-Neumann (DtN) maps associated to the linearized potential equation for the complement of the sub-domains $\Omega \backslash \Omega_{i}$ and $\Omega \backslash \Omega_{j}$ [14]. The definition and possible approximations of these DtN maps are detailed in the following section. By summing Eqs. (5a) and (5b), one obtains:

$$
\mathcal{L}_{i \rightarrow j} \phi_{i}+\mathcal{L}_{j \rightarrow i} \phi_{j}=\rho_{0}\left(1-M_{n_{i}}^{2}\right)\left(\mathcal{S}_{i} \phi_{i}+\mathcal{S}_{j} \phi_{j}\right) .
$$




\subsection{Approximation of the DtN map}

In a smooth domain $\Omega$, the DtN map links the value of the field $\phi$ on a boundary $\Gamma$ with its normal derivative:

$$
\frac{\partial \phi}{\partial n}=\mathcal{S} \phi, \quad \text { on } \Gamma .
$$

However, this operator is often non-local and is cumbersome to implement in a finite element strategy since it would break the sparsity of the global matrix. The simplest approach is to use a zeroth order approximation of the DtN map for the linearized potential equation. It amounts to coupling the sub-domains via a Robin-type boundary condition, i.e.:

$$
\mathcal{S}_{i}=-\frac{\mathrm{i} k_{0}}{1+M_{n_{i}}}, \quad \text { on } \partial \Omega_{i}, \quad \mathcal{S}_{j}=-\frac{\mathrm{i} k_{0}}{1+M_{n_{j}}}, \quad \text { on } \partial \Omega_{j}
$$

Higher order local approximations could be derived through the theoretical framework of microlocal analysis such as initiated in [17]. Preliminary analysis shows that the DtN operator for the linearized potential equation is of the same nature as for Helmholtz problems and can be approximated by a first-order pseudo-differential operator of square-root type. A detailed analysis of the DtN map and its approximations for the linearized potential equation is currently under investigation and will be the topic of a future work. Let us consider the second-order Taylor expansion of the square-root operator for a flat boundary. It reads:

$$
\mathcal{S}_{i}=-\frac{\mathrm{i} k_{0}}{1+M_{n_{i}}}-\frac{\mathrm{i}}{2 k_{0}} \Delta_{\Gamma}, \quad \text { on } \partial \Omega_{i}, \quad \mathcal{S}_{j}=-\frac{\mathrm{i} k_{0}}{1+M_{n_{j}}}-\frac{\mathrm{i}}{2 k_{0}} \Delta_{\Gamma}, \quad \text { on } \partial \Omega_{j},
$$

where $\Delta_{\Gamma}$ denotes the surface Laplace-Beltrami operator. These transmission operators or interface conditions are valid in the high frequency limit for propagative modes. Contrary to the exact DtN map, the conditions are now local and can be directly implemented using the available finite element matrices. Evanescent modes can be damped by introducing a rotation angle $\alpha \in[0,-\pi]$ in the Taylor expansion, which corresponds to rotating the branch-cut of the square-root operator away from the negative half-plane [18]. The zeroth order condition (8) becomes

$$
\mathcal{S}_{i}^{\alpha}=-\mathrm{i} k_{0}\left(\frac{\mathrm{e}^{\mathrm{i} \alpha / 2}-M_{n_{i}}}{1-M_{n_{i}}^{2}}\right), \quad \mathcal{S}_{j}^{\alpha}=-\mathrm{i} k_{0}\left(\frac{\mathrm{e}^{\mathrm{i} \alpha / 2}-M_{n_{j}}}{1-M_{n_{j}}^{2}}\right),
$$

and the second-order one (9) reads:

$$
\mathcal{S}_{i}^{\alpha}=-\mathrm{i} k_{0}\left(\frac{\cos (\alpha / 2)-M_{n_{i}}}{1-M_{n_{i}}^{2}}\right)-\mathrm{i} \frac{\mathrm{e}^{-\mathrm{i} \alpha / 2}}{2 k_{0}} \Delta_{\Gamma}, \quad \mathcal{S}_{j}^{\alpha}=-\mathrm{i} k_{0}\left(\frac{\cos (\alpha / 2)-M_{n_{j}}}{1-M_{n_{j}}^{2}}\right)-\mathrm{i} \frac{\mathrm{e}^{-\mathrm{i} \alpha / 2}}{2 k_{0}} \Delta_{\Gamma} .
$$

The choice $\alpha=0$ corresponds to the damping of propagative modes whereas $\alpha=-\pi$ corresponds to evanescent modes. This angle can be tuned in order to find an optimal balance between the damping of propagative and evanescent modes.

\subsection{Introduction of the interface unknowns}

Two interface unknowns are introduced to represent the forcing generated by a sub-domain on its neighbors. These dual variables are defined by

$$
\lambda_{j \rightarrow i}=\mathcal{L}_{j \rightarrow i} \phi_{j}, \quad \lambda_{i \rightarrow j}=\mathcal{L}_{i \rightarrow j} \phi_{i} .
$$

The boundary integrals from variational formulations (2) and (3) become:

$$
\begin{aligned}
& \int_{\Gamma_{i j}} \rho_{0} \overline{\psi_{i}}\left[\mathrm{i} k_{0} M_{n_{i}} \phi_{i}+M_{n_{i}} M_{\tau_{i}} \frac{\partial \phi_{i}}{\partial \tau_{i}}+\left(M_{n_{i}}^{2}-1\right) \mathcal{S}_{i} \phi_{i}+\frac{\lambda_{j \rightarrow i}}{\rho_{0}}\right] \mathrm{d} \Gamma, \\
& \int_{\Gamma_{i j}} \rho_{0} \overline{\psi_{j}}\left[\mathrm{i} k_{0} M_{n_{j}} \phi_{j}+M_{n_{j}} M_{\tau_{j}} \frac{\partial \phi_{j}}{\partial \tau_{j}}+\left(M_{n_{j}}^{2}-1\right) \mathcal{S}_{j} \phi_{j}+\frac{\lambda_{i \rightarrow j}}{\rho_{0}}\right] \mathrm{d} \Gamma .
\end{aligned}
$$

Since we have introduced two additional fields, defined only on $\Gamma_{i j}$, we have to derive two supplementary equations which are actually provided by (6):

$$
\lambda_{j \rightarrow i}+\lambda_{i \rightarrow j}=\rho_{0}\left(1-M_{n_{i}}^{2}\right)\left(\mathcal{S}_{i}+\mathcal{S}_{j}\right) \phi_{i}, \quad \text { on } \Gamma_{i j},
$$




$$
\lambda_{j \rightarrow i}+\lambda_{i \rightarrow j}=\rho_{0}\left(1-M_{n_{j}}^{2}\right)\left(\mathcal{S}_{i}+\mathcal{S}_{j}\right) \phi_{j}, \quad \text { on } \Gamma_{i j} .
$$

They are solved by using Galerkin formulations:

$$
\begin{aligned}
& \int_{\Gamma_{i j}} \overline{\mu_{j \rightarrow i}} \lambda_{j \rightarrow i}+\overline{\mu_{j \rightarrow i}} \lambda_{i \rightarrow j} \mathrm{~d} S-\int_{\Gamma_{i j}} \rho_{0}\left(1-M_{n_{i}}^{2}\right) \overline{\mu_{j \rightarrow i}}\left(\mathcal{S}_{i}+\mathcal{S}_{j}\right) \phi_{i} \mathrm{~d} S=0, \\
& \int_{\Gamma_{i j}} \overline{\mu_{i \rightarrow j}} \lambda_{j \rightarrow i}+\overline{\mu_{i \rightarrow j}} \lambda_{i \rightarrow j} \mathrm{~d} S-\int_{\Gamma_{i j}} \rho_{0}\left(1-M_{n_{j}}^{2}\right) \overline{\mu_{i \rightarrow j}}\left(\mathcal{S}_{i}+\mathcal{S}_{j}\right) \phi_{j} \mathrm{~d} S=0 .
\end{aligned}
$$

One can now use the transmission operators $\mathcal{S}_{i}$ and $\mathcal{S}_{j}$ to obtain a system of four Galerkin formulations to discretize. For example, the second-order condition (9) applied to the interface formulation (16) gives:

$$
\int_{\Gamma_{i j}} \overline{\mu_{j \rightarrow i}} \lambda_{j \rightarrow i}+\overline{\mu_{j \rightarrow i}} \lambda_{i \rightarrow j} \mathrm{~d} S=-2 \mathrm{i} k_{0} \int_{\Gamma_{i j}} \rho_{0} \overline{\mu_{j \rightarrow i}} \phi_{i} \mathrm{~d} S+\frac{\mathrm{i}}{k_{0}} \int_{\Gamma_{i j}} \rho_{0}\left(1-M_{n_{i}}^{2}\right) \overline{\nabla_{\Gamma} \mu_{j \rightarrow i}} \cdot \nabla_{\Gamma} \phi_{i} \mathrm{~d} S,
$$

where $\nabla_{\Gamma}$ denotes the gradient operator along the surface $\Gamma$. In deriving the above expression we have assumed that there is no contribution from the contour term introduced by the integration by parts. We do not use any particular treatment for cross-points or corners. Such a treatment is expected to represent more accurately the DtN map and to further accelerate the convergence of the domain decomposition algorithm [19].

\subsection{Interface problem}

Discretizing the variational formulations using a finite element method (see Section 4 for more details) yields an algebraic system of the form:

$$
\begin{aligned}
& \mathbf{K}_{i} \boldsymbol{\phi}_{i}+\mathbf{B}_{j i} \mathbf{M}_{i j} \lambda_{j \rightarrow i}=\mathbf{f}_{i}, \\
& \mathbf{K}_{j} \boldsymbol{\phi}_{j}+\mathbf{B}_{i j} \mathbf{M}_{i j} \lambda_{i \rightarrow j}=\mathbf{f}_{j},
\end{aligned}
$$

where the vector $\boldsymbol{\phi}_{i}$ contains the $N_{i}$ degrees of freedom for the solution in sub-domain $\Omega_{i}$. The vector $\lambda_{j \rightarrow i}$ stores the $N_{i j}$ degrees of freedom for the Lagrange multiplier on the interface $\Gamma_{i j}$. The sub-domain matrices $\mathbf{K}_{i}$ contain the usual terms associated with the discrete form of the linearized potential equation. The matrices $\mathbf{B}_{i j}$ are boolean of size $N_{j} \times N_{i j}$, while $\mathbf{M}_{i j}$ designates the mass matrix. The right hand side $\mathbf{f}_{i}$ represents a source term such as an acoustic monopole or a modal source.

Similarly the additional variational formulations (16) and (17) yield

$$
\begin{aligned}
& \mathbf{M}_{i j} \lambda_{i \rightarrow j}+\mathbf{M}_{i j} \lambda_{j \rightarrow i}+\mathbf{N}_{i j} \mathbf{B}_{j i}^{T} \boldsymbol{\phi}_{i}=\mathbf{0}, \\
& \mathbf{M}_{i j} \lambda_{i \rightarrow j}+\mathbf{M}_{i j} \lambda_{j \rightarrow i}+\mathbf{N}_{i j} \mathbf{B}_{i j}^{T} \boldsymbol{\phi}_{j}=\mathbf{0},
\end{aligned}
$$

where $\mathbf{N}_{i j}$ defines the terms associated with the interface condition. Eqs. (19) and (20) can be used to eliminate the degrees of freedom $\boldsymbol{\phi}_{i}$ in each sub-domain:

$$
\boldsymbol{\phi}_{i}=\mathbf{K}_{i}^{-1} \mathbf{f}_{i}+\mathbf{K}_{i}^{-1} \mathbf{B}_{j i} \mathbf{M}_{i j} \lambda_{j \rightarrow i}, \quad \boldsymbol{\phi}_{j}=\mathbf{K}_{j}^{-1} \mathbf{f}_{j}-\mathbf{K}_{j}^{-1} \mathbf{B}_{i j} \mathbf{M}_{i j} \lambda_{i \rightarrow j},
$$

where the LU factorization of the matrices $\mathbf{K}_{i}$ are calculated using a direct solver. The above expression can then be used to eliminate the degrees of freedom $\boldsymbol{\phi}_{i}$ from Eqs. (21) and (22) to obtain the so-called interface problem:

$$
\left[\begin{array}{cc}
\mathbf{M}_{i j} & \mathbf{M}_{i j}+\mathbf{N}_{i j} \mathbf{B}_{j i}^{T} \mathbf{K}_{i}^{-1} \mathbf{B}_{i j} \mathbf{M}_{i j} \\
\mathbf{M}_{i j}+\mathbf{N}_{i j} \mathbf{B}_{i j}^{T} \mathbf{K}_{j}^{-1} \mathbf{B}_{j i} \mathbf{M}_{i j} & \mathbf{M}_{i j}
\end{array}\right]\left(\begin{array}{c}
\lambda_{j \rightarrow i} \\
\lambda_{i \rightarrow j}
\end{array}\right)=\left(\begin{array}{c}
\mathbf{N}_{i j} \mathbf{B}_{j i}^{T} \mathbf{K}_{i}^{-1} \mathbf{f}_{i} \\
\mathbf{N}_{i j} \mathbf{B}_{i j}^{T} \mathbf{K}_{j}^{-1} \mathbf{f}_{j}
\end{array}\right),
$$

which involves only the degrees of freedom $\lambda_{j \rightarrow i}$ defined on the interfaces between sub-domains, and is therefore of a much smaller size than the global system written for the primal variables $\boldsymbol{\phi}_{i}$.

It is possible to simplify the definition of the interface problem by defining $\tilde{\lambda}_{j \rightarrow i}=\mathbf{M}_{i j} \lambda_{j \rightarrow i}$ which leads to:

$$
\left[\begin{array}{cc}
\mathbf{I} & \mathbf{I}+\mathbf{N}_{i j} \mathbf{B}_{j i}^{T} \mathbf{K}_{i}^{-1} \mathbf{B}_{i j} \\
\mathbf{I}+\mathbf{N}_{i j} \mathbf{B}_{i j}^{T} \mathbf{K}_{j}^{-1} \mathbf{B}_{j i} & \mathbf{I}
\end{array}\right]\left(\begin{array}{l}
\tilde{\lambda}_{j \rightarrow i} \\
\tilde{\lambda}_{i \rightarrow j}
\end{array}\right)=\left(\begin{array}{l}
\mathbf{N}_{i j} \mathbf{B}_{j i}^{T} \mathbf{K}_{i}^{-1} \mathbf{f}_{i} \\
\mathbf{N}_{i j} \mathbf{B}_{i j}^{T} \mathbf{K}_{j}^{-1} \mathbf{f}_{j}
\end{array}\right) .
$$

This is exactly the discretization of a fixed point system resulting from the additive Schwarz domain decomposition algorithm. It is solved iteratively using a Krylov method such as GMRES or ORTHODIR [20]. The matrix on the 
left-hand side of Eq. (23) is not formed since for these iterative solvers one only has to perform matrix-vector products. We note that the use of different interface conditions $\mathcal{S}_{i}$ and $\mathcal{S}_{j}$ modify the matrices $\mathbf{K}_{i}$ and $\mathbf{N}_{i j}$. It can be seen as a preconditioner for the iterative procedure.

\section{Adaptive high-order finite elements}

The domain decomposition formulation is discretized using a $p$-FEM high-order finite element method [21,22]. Each sub-domain $\Omega_{i}$ is approximated by a tessellation of non-overlapping elements. On each element the approximation of the solution $\phi(\mathbf{x}, t)=\Phi(\mathbf{x}) e^{\mathrm{i} \omega t}$ is constructed as a linear combination of high-order shape functions $w_{n}(\mathbf{x})$ :

$$
\Phi(\mathbf{x})=\sum_{n=1}^{N} \Phi_{n} w_{n}(\mathbf{x}),
$$

where the degrees of freedom $\Phi_{n}$ are the complex coefficients of each shape function. The Lobatto shape functions are used in this work [23]. There are four different types of shape functions. The standard linear shape functions whose associated degrees of freedom are the values of the solution at the nodes of the elements. Face and edge functions are defined on the corresponding geometrical entity. The fourth category are the so-called 'bubble' shape functions which vanish on the boundary of each element. Apart from the linear shape functions which are nodal all the others are modal because the associated degrees of freedom do not correspond to the solution at specific points on the element. The integration on the reference element is done by a tensorized Gauss-Legendre quadrature rule.

After calculating and assembling the matrices from all elements, one is left with a sparse linear system of equations which is solved using a fast, parallel multi-frontal solver [24,25].

There are several benefits in using the Lobatto shape functions. They have been shown to provide good performance and conditioning [26]. Furthermore their 'hierarchic' nature implies that the shape functions at order $p$ form a subset of the shape functions at order $p+1$. This reduces the assembling time of the global sparse matrix when different shape function orders are used throughout the mesh. Another useful property is that it is possible to eliminate the degrees of freedom associated with the bubble shape functions. This technique, called static condensation, allows to reduce the size of the linear system before it is formed and solved, hence reducing the memory footprint of the method.

With this method it is possible to adjust the order of interpolation in each element. This is particularly useful for several reasons. Firstly, when applying the standard finite element method for problems with strongly non-uniform flows it is necessary to refine the mesh in the region where the mean flow velocity is high since the acoustic wavelength can be significantly reduced by the convective effect. Secondly, typical use cases of these methods involves performing not one but several calculations each at a different frequency. One has to generate several meshes to accommodate the different mesh resolution requirements for each frequency which is time consuming for the user. Another option is to generate a single fine mesh capable of resolving the complete range of frequencies, which drastically increases the time and computational resources needed for the analysis.

The use of a variable polynomial order in each element provides a way to address these two issues. The order is defined thanks to an a priori error indicator introduced in [21] and extended in [22] to the linearized potential equation. The error is estimated on a single one-dimensional element based on the local mesh resolution. The appropriate order is then selected from tabulated values for a prescribed target accuracy $E_{T}$. This simple a priori rule has shown to be robust and ensures that the numerical error on each element is controlled in terms of the element size, frequency and flow properties. In this way, the mesh does not need to be explicitly refined by the user, since the polynomial order will be automatically increased in region where the flow velocity is high. When performing several calculations for a series of frequencies, there is no need to prepare several meshes because the adaptive scheme will progressively increase the polynomial order for high frequencies, thus ensuring that the same level of accuracy is achieved for all results.

\section{Cylinder scattering in a potential flow}

\subsection{Verification}

The test case used to validate the approach and implementation consists in the scattering of a point source by a rigid cylinder. It is placed in a steady, two-dimensional, incompressible, inviscid and irrotational flow such that far 
Table 1

Validation of the surrounding perfectly matched layer. Relative errors (in \%) in the 2-norm and infinity-norm on the PML interface between the reference $\left(\mathrm{L}_{\mathrm{PML}}=\right.$ $40 h)$ and approximate $\left(\mathrm{L}_{\mathrm{PML}}=h\right)$ solutions for three frequencies. The numerical parameters are described below.

\begin{tabular}{llll}
\hline & $\omega=10$ & $\omega=25$ & $\omega=40$ \\
\hline$L^{2}$ error & 2.27 & 1.49 & 1.35 \\
$L^{\infty}$ error & 2.83 & 2.13 & 3.48 \\
\hline
\end{tabular}

enough from the cylinder, the velocity $V_{\infty}$ is uniform and aligned with the $x$-axis. The expression of the velocity can be derived using the Laplace equation for its potential. In cylindrical coordinates $(r, \theta)$, it is given by:

$$
v_{r}(r, \theta)=V_{\infty}\left(1-\frac{R^{2}}{r^{2}}\right) \cos \theta, \quad v_{\theta}(r, \theta)=-V_{\infty}\left(1+\frac{R^{2}}{r^{2}}\right) \sin \theta,
$$

where $R$ is the radius of the cylinder. The flow has zero velocities at the stagnation points $(r=R$ and $\theta=0,2 \pi)$ and a maximum velocity corresponding to the upper and lower surface of the cylinder $(r=R$ and $\theta=\pi / 2,3 \pi / 2)$. In the computations performed here, the potential mean flow is prescribed analytically. The speed of sound $c_{0}$ and the density $\rho_{0}$ are set to unity. We use $R=1, V_{\infty}=-0.4$. The resulting mean flow direction and magnitude is plotted in Fig. 1.

The location and amplitude of the point source are $\left(x_{s}, y_{s}\right)=(-R-0.4,0)$ and $A=1$, respectively. The acoustic field is solved on a square domain of dimension $[-2 R, 2 R] \times[-2 R, 2 R]$ containing the cylinder which is centered at the origin. A hard wall boundary condition is imposed at the surface of the cylinder and a Perfectly Matched Layer of width $\mathrm{L}_{\mathrm{PML}}=h$ is used to absorb the waves that may be reflected back into the numerical domain [12]. The latter is verified in Table 1 with a reference solution based on a thicker PML, which was proven to converge towards the physical solution [27]. Spurious wave reflections are at most 3.5\% locally and $2.3 \%$ globally. Although not perfect, the proposed narrow PML domain is considered to be a reasonable approximation of a non-reflecting boundary. A more accurate PML could be set up by either increasing its width or resorting to specific techniques for convected problems [28,27,29].

An unstructured mesh of quadratic triangular elements is used to represent the computational domain (see Fig. 1). The mesh is refined close to the surface of the cylinder. Compared to the typical mesh size $h$ at the outer boundary, the mesh size at the surface of the cylinder is set to $h / 3$. The same refinement is used around the point source where the solution is expected to be singular.

In the absence of analytic solution, we compare the solution obtained with the optimized Schwarz method to the solution produced using the same mesh and a direct solver. The mesh is split into sub-domains thanks to an automatic graph partitioner, namely the METIS library [30]. The polynomial order distribution used for the hybrid and direct solvers are identical for a given problem.

The problem is considered at three different frequencies for a given number of sub-domains: $\omega=10,25$ and 100 for a target accuracy of $E_{T}=1 \%$ on the $L^{2}$ error. The iterative procedure is stopped when the interface residual reaches $r_{I}=1 \mathrm{e}-8$. Accounting for the convective effect induced by the mean flow, these frequencies correspond respectively to wave number magnitudes of approximately 50,125 and 500 in the regions where the mean flow magnitude is the largest. For each frequency, the typical mesh size is chosen such that the shortest wavelength at the surface of the cylinder is described by at least 6 nodes in order to control the geometrical error.

The solutions in the domain are given in Fig. 2 for $N_{s}=9$ sub-domains along with the real part of the solutions at each frequency on the perimeter of the cylinder $($ at $r=R)$. While large wavelengths are found in the region downstream of the source, short creeping upstream waves are observed along the cylinder. We see that there is an excellent agreement between the solutions, even at high frequency and in the most oscillatory regions corresponding to $\theta= \pm \pi / 2$.

\subsection{Performance of the interface conditions}

The performance of the different transmissions operators presented in Section 3.2 are now compared for a given set of numerical parameters. We want to study the accuracy of the operators $\mathcal{S}_{i}$ and $\mathcal{S}_{j}$ compared to the solution 

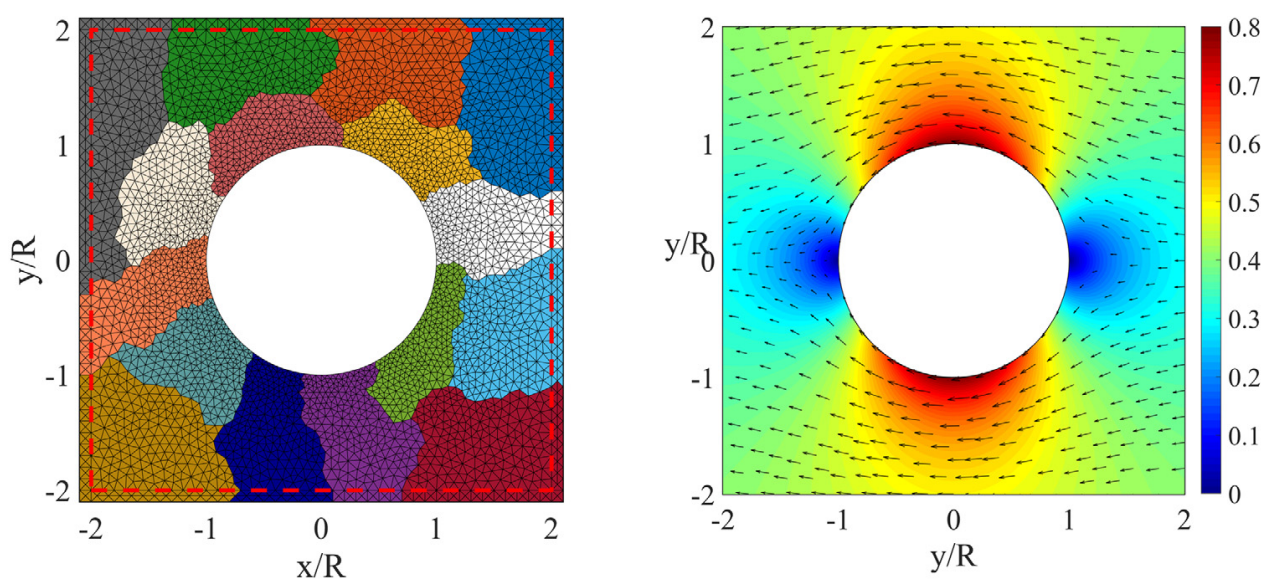

Fig. 1. Left: Example of partitioning of the discretized domain with $N_{s}=16$ sub-domains. The physical and PML regions are delimited by the red dashed line. Right: Magnitude of the mean flow velocity field around the cylinder.

that would have been obtained by a direct solver. The resulting remaining error is referred to as global residual. We define it as the average residual over the sub-domains volumic systems

$$
r_{g}=\frac{1}{N_{s}} \sum_{i=1}^{N_{s}} \frac{\left\|\mathbf{K}_{i} \boldsymbol{\phi}_{i}-\mathbf{f}_{i}\right\|_{2}}{\left\|\mathbf{f}_{i}\right\|_{2}},
$$

where $\|\cdot\|_{2}$ refers to the Euclidian norm. The interface residual is always verified to decrease consistently, but depends on the selected interface condition.

In Fig. 3, the number of iterations required to reach a global residual of $r_{g}=1 \mathrm{e}-6$ is plotted as a function of the rotation angle $\alpha$. For both conditions of order 0 and 2, there is an angle for which the number of iterations is minimal. Physically, this angle corresponds to the optimal balance between the attenuation of propagative and evanescent modes hitting the interfaces. The global residual for the second-order condition decreases faster than the zeroth order condition. This confirms that the DtN map is better approximated thanks to a higher order Taylor expansion. The second-order condition equipped with the optimal rotation angle further improves the approximation since the evanescent modes are captured. This behavior is confirmed by the eigenvalues distribution shown in Fig. 4. In fact, the second-order condition drives away the eigenvalues that accumulate around zero real part, thus improving the conditioning of the interface problem. The rotation angle plays a different role. It moves the eigenvalues lying on the unit circle towards the point $(1,0)$, thus leading to a better clustering for the Krylov method. These eigenvalues correspond to the evanescent modes on the sub-domain interfaces. Similar behavior has been observed and analyzed for Maxwell's equations in [8].

However, the spreading of the eigenvalues in the complex plane suggests that the presented conditions are only sub-optimal. In this case, the second-order condition (11) leads to a gain of a factor 4 in terms of iterations compared to the Robin-type condition (8).

\subsection{Scalability}

We would like to examine now the performance of the method in view of industrial applications. The robustness of the method with respect to the frequency and the number of sub-domains is analyzed for that purpose. The so-called scalability results are gathered in Table 2.

One way to analyze the high frequency behavior of the interface conditions is to keep the numerical error under control through the frequency sweep. Let us consider the number of degrees of freedom per shortest wavelength as defined in [31]:

$$
d_{\lambda}^{*}=\frac{2 \pi P}{\omega h}\left(1-\left|M_{\max }\right|\right),
$$



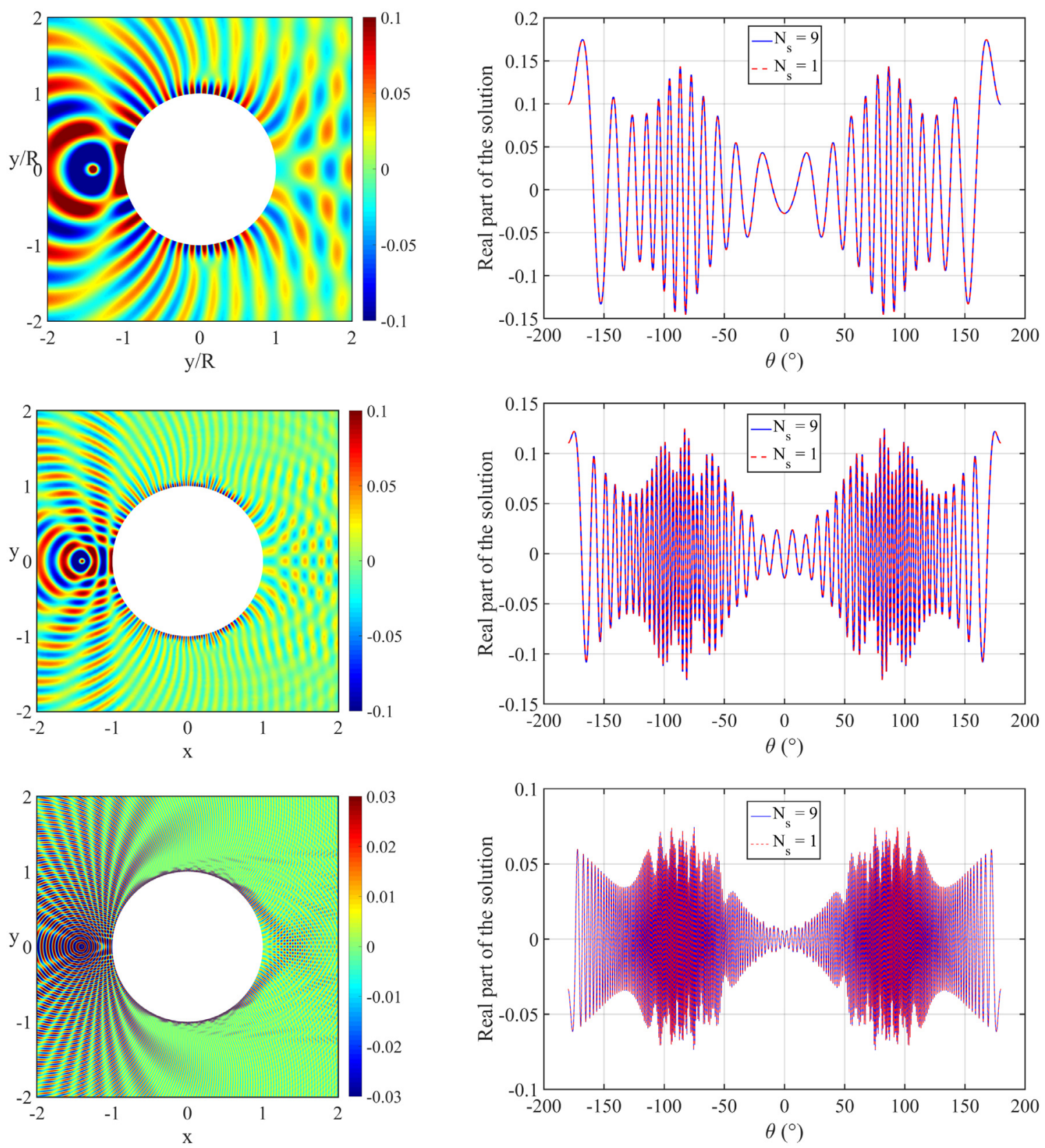

Fig. 2. Example of numerical solutions obtained by domain decomposition for $\omega=10, \omega=25$ and $\omega=100$. Left: real part of the pressure field. Right: directivity of the solution along the perimeter of the cylinder for $N_{s}=1$ and $N_{s}=9$.

where $P$ is the polynomial order of the shape functions and $M_{\max }$ the maximum value of the local Mach number in the domain. In this section only, we fix the polynomial order of the elements. While the frequency is increased in the experiments, we maintain a value of $d_{\lambda}^{*}=5$ by adjusting the mesh size. This strategy allows to keep the interpolation and dispersion errors constant in the domain and to have a better control on the pollution effect during the sweep.

Table 2 indicates that high oscillatory problems are more easily tackled by the second-order condition. The number of iterations needed to reach $r_{g}=1 \mathrm{e}-6$ increases with the frequency by a faster rate for the zeroth order condition than for the second-order one. As previously mentioned, this is the consequence of a more accurate 

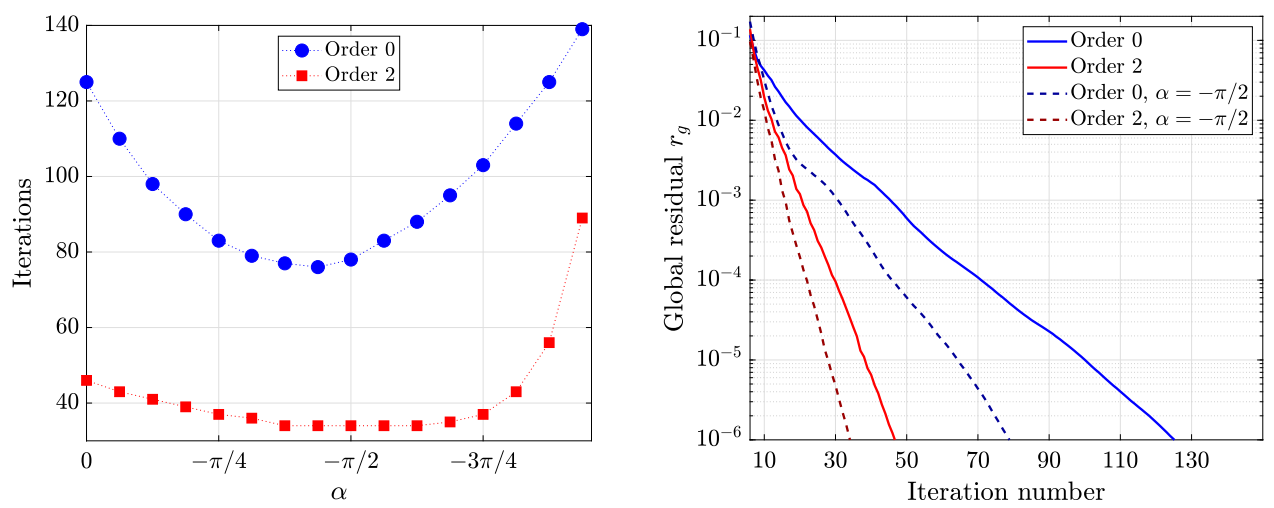

Fig. 3. Left: Number of iterations required to reach $r_{g}=1 \mathrm{e}-6$ as a function of the rotation angle $\alpha$. Right: evolution of the global residual for the interface conditions of order 0 and 2. Numerical parameters: $V_{\infty}=-0.4, \omega=25, N_{s}=8, h / R=0.06$ and $E_{T}=0.5 \%$.

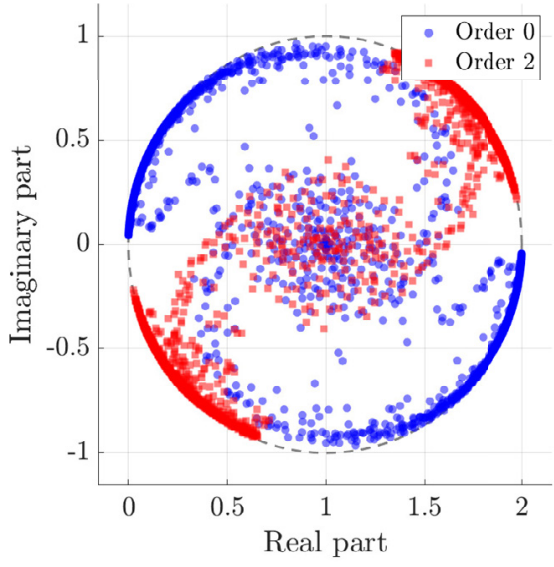

(a) $\alpha=0$

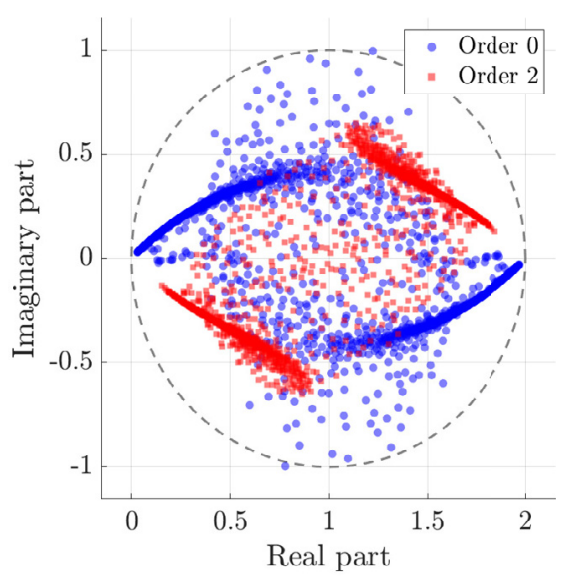

(b) $\alpha=-\pi / 2$

Fig. 4. Eigenvalues distribution for the interface problem for two rotation angles $\alpha$. The numerical parameters are those of Fig. 3 .

approximation of the DtN map. The optimal interface condition would converge independently of the frequency. Improvements towards this goal have been achieved with Padé-type transmission boundary conditions. They only require a small amount of additional cost [4]. The rotation angle acts on the evanescent part of the spectrum and does not significantly affect the frequency scalability properties for both conditions of order 0 and 2 .

The number of sub-domains is then increased by fixing the other numerical parameters. The total number of degrees of freedom on the interfaces $N_{D O F}^{I}$ is also reported. As expected from previous studies for Helmholtz problems [16], the method does not scale with the number of sub-domains. This can be understood since the information exchanged between the sub-domains is only local. The use of coarse spaces [32], shifted Laplacian [33] or sweeping preconditioners [34-36] would help to retrieve parallel efficiency by global information exchange. It is an active area of research [37].

\section{Application to a turbofan intake}

\subsection{The turbofan model}

The proposed methodology is now applied to solve a problem of industrial relevance: the sound radiation from a generic turbofan intake. Detailed information concerning this test case are available in Prinn [26] and Mustafi [38]. This geometry was previously used to benchmark the performance of the a priori $p$-adaptive strategy for flow acoustics in $[22,39]$. 
Table 2

Number of iterations required to reach a global residual $r_{g}=1 \mathrm{e}-6$ for different number of sub-domains, frequencies and interface conditions. Numerical parameters: $V_{\infty}=-0.4, d_{\lambda}^{*}=5, P=4$. O0: order $0, \mathrm{O} 2$ : order 2 . The rotation angle is set to $-\pi / 2$ for the condition $\mathrm{O} 2, \alpha$.

\begin{tabular}{|c|c|c|c|c|c|c|c|c|c|c|c|c|}
\hline \multirow[b]{2}{*}{$N_{s}$} & \multicolumn{4}{|c|}{$\omega=10$} & \multicolumn{4}{|c|}{$\omega=20$} & \multicolumn{4}{|c|}{$\omega=40$} \\
\hline & $N_{D O F}^{I}$ & $\mathrm{O} 0$ & $\mathrm{O} 2$ & $\mathrm{O} 2, \alpha$ & $N_{D O F}^{I}$ & $\mathrm{O} 0$ & $\mathrm{O} 2$ & $\mathrm{O} 2, \alpha$ & $N_{D O F}^{I}$ & $\mathrm{O} 0$ & $\mathrm{O} 2$ & $\mathrm{O} 2, \alpha$ \\
\hline 2 & 260 & 95 & 32 & 23 & 476 & 103 & 34 & 25 & 948 & 131 & 43 & 30 \\
\hline 4 & 616 & 123 & 42 & 30 & 1016 & 129 & 43 & 30 & 1872 & 164 & 45 & 32 \\
\hline 8 & 1320 & 126 & 48 & 34 & 2312 & 148 & 46 & 33 & 4728 & 170 & 50 & 36 \\
\hline 16 & 2348 & 160 & 70 & 49 & 4276 & 172 & 69 & 47 & 8504 & 185 & 77 & 54 \\
\hline 32 & 3698 & 179 & 89 & 62 & 6694 & 183 & 85 & 59 & 13426 & 195 & 92 & 62 \\
\hline
\end{tabular}

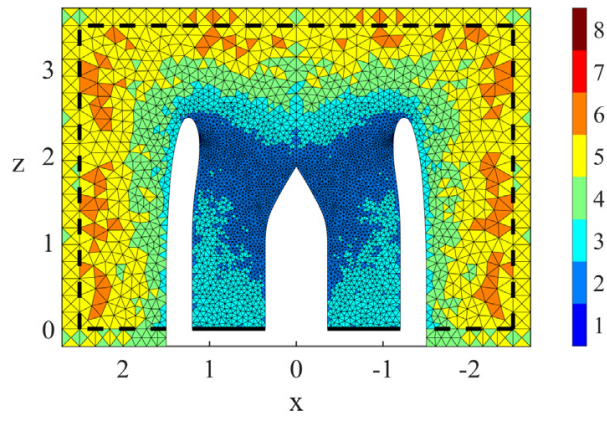

(a) Approach

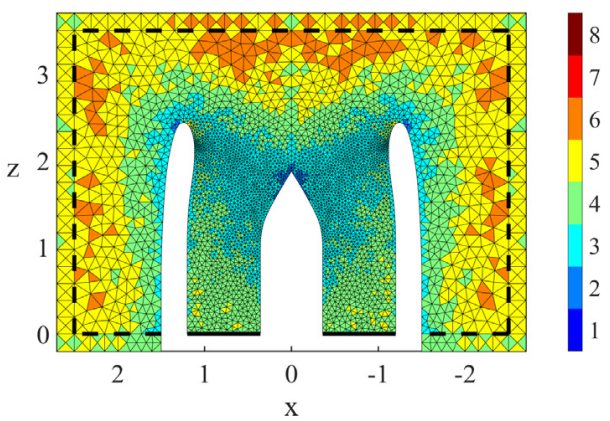

(b) Sideline

Fig. 5. Finite elements polynomial orders on the $x-z$ plane. The physical and PML regions are delimited by the black dashed line. The location of the input boundary condition is highlighted by the black solid line.

We focus here on the approach and sideline flight configurations, used for the noise certification of aeroengines. They differ from the Mach number at the fan face, being $M_{f}=0.22$ for the approach case and $M_{f}=0.55$ for the sideline case. The conditions at infinity are as follows: sound speed $c_{\infty}=340 \mathrm{~m} / \mathrm{s}$, density $\rho_{\infty}=1.2 \mathrm{~kg} / \mathrm{m}^{3}$ and Mach number $M_{\infty}=u_{\infty} / c_{\infty}=0.25$. The background mean flow used for the acoustic simulation is shown in Fig. 3(c) of Ref. [22]. It was obtained using a potential, compressible, inviscid flow model. The input boundary condition consists in a single rotor-locked mode at the Blade Passing Frequency (BPF) of the fan $f=1300 \mathrm{~Hz}$. It is enforced by a surface integral on the fan plane boundary. Results are presented here for the first radial mode with azimuthal order 24. The computational domain is a cylinder of length $3.5 \mathrm{~m}$ and radius $2.5 \mathrm{~m}$. A cylindrical Perfectly Matched Layer is used to efficiently model the outgoing waves [13]. The remaining boundaries, namely the nacelle and spinner of the intake, are set to be perfectly reflecting walls. The mesh consists of conventional quadratic, 10-nodes, tetrahedral Lagrange elements, with a characteristic length of $h=20 \mathrm{~cm}$ in the far-field and $h=4$ $\mathrm{cm}$ close to the walls of the nacelle. The adaptive procedure referred in Section 4 is applied to assign the orders in each element across the mesh with a target accuracy of $E_{T}=5 \%$ on the $L^{2}$ error. The mesh is then split into $N_{s}=6$ sub-domains using the METIS library [30]. In order to take into account the element order distribution in the partitioning and to guarantee a good repartition of the degrees of freedom between the different sub-domains, each element is weighted with a factor $p_{e}^{3}$, where $p_{e}$ designates the local element order. In the following computations, the element orders range from $p=1$ to $p=8$ depending on the mean flow magnitude and the mesh size. Fig. 5 shows the elements order repartition and the location of the boundary conditions. The variation of the element orders in the nacelle is mainly due to the mean flow magnitude contrast between the two configurations.

\subsection{Numerical procedure and results}

A parallel implementation of the ORTHODIR algorithm is used to solve iteratively the interface problem (23). One MPI process is assigned to each sub-domain, and each MPI process is allocated to a separate node on a HPC cluster with InfiniBand interconnect. The most computationally demanding task for each process involves the LU factorization of the linear system for each sub-domain which is performed by the MUMPS solver version 
Table 3

Characteristics of the domain decomposition sub-domain matrices after static condensation. Minimum, maximum and total data over the $N_{s}=6$ sub-domains.

\begin{tabular}{|c|c|c|c|c|c|c|}
\hline \multirow[t]{2}{*}{ Configuration } & \multicolumn{3}{|l|}{ Approach } & \multicolumn{3}{|l|}{ Sideline } \\
\hline & Min & Max & Total & Min & Max & Total \\
\hline Degrees of freedom & 520521 & 651205 & 3396321 & 872971 & 1048958 & 5770072 \\
\hline Non-zeros & 36524882 & 69631702 & 342187703 & 76632678 & 112184616 & 563898984 \\
\hline LU memory & $17 \mathrm{~Gb}$ & $23 \mathrm{~Gb}$ & $117 \mathrm{~Gb}$ & $34 \mathrm{~Gb}$ & $46 \mathrm{~Gb}$ & $241 \mathrm{~Gb}$ \\
\hline
\end{tabular}

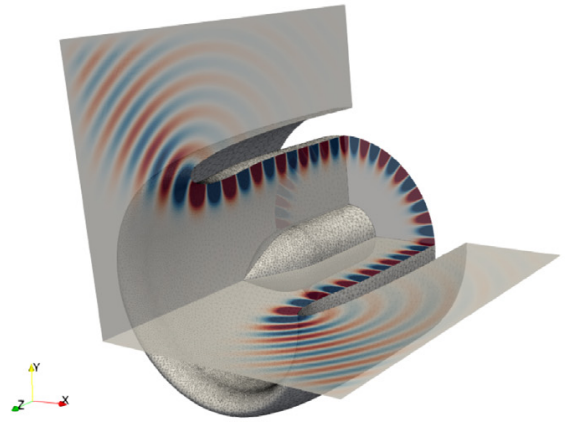

(a) Approach

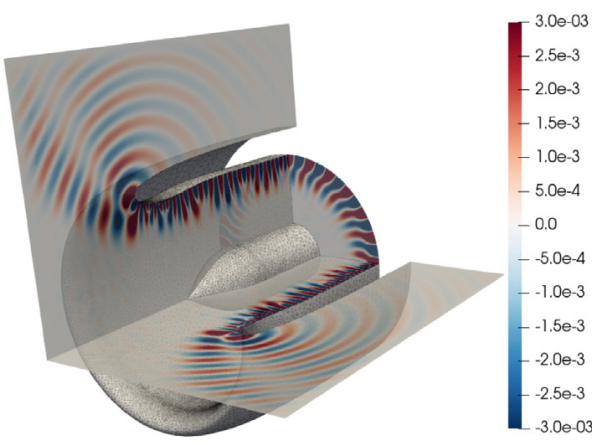

(b) Sideline

Fig. 6. Real part of the acoustic potential obtained for the mode $(24,1)$ at the first BPF $(f=1300 \mathrm{~Hz})$ for two static flow configurations. The solutions were obtained with $N_{s}=6$ sub-domains.

5.1.2 [24,25]. In the sideline configuration, the largest matrix to factorize had roughly 1 million degrees of freedom, which was factorized using $46 \mathrm{~Gb}$ of memory. The smallest matrix had 873 thousand degrees of freedom and required $38 \mathrm{~Gb}$ of memory, which indicates a relatively good load balancing between the different processes. The approach configuration required less resources, namely between 17 and $23 \mathrm{~Gb}$ of memory. Table 3 summarizes the computational characteristics of the two simulations. The interface problem contains for the approach and sideline cases respectively 48684 and 50024 Lagrange multipliers.

Fig. 6 presents the real part of the acoustic potential for the two mean flow configurations. The mesh is refined near the throat of the inlet where the Mach number exceeds 0.8 in the sideline configuration. This induces a significant shortening of the waves in that region, which is well captured by the high-order adaptive finite element model.

Fig. 7 shows the interface residual of the iterative procedure for the zeroth and second-order interface conditions. The second-order condition is equipped with a rotation angle of $\alpha=-\pi / 2$. The interface residual to reach convergence is set to $r_{I}=1 \mathrm{e}-8$. The global residual defined in (25) is computed when the required interface residual is reached. With the zeroth order condition, the approach and sideline configurations converged respectively in 404 and 830 iterations to a global residual of $1.8 \mathrm{e}-7$ and $1.1 \mathrm{e}-6$. The iterations reduce respectively to 109 and 435 to a global residual of $4.8 \mathrm{e}-9$ and $5.7 \mathrm{e}-9$ with the second-order condition. The convergence speed up is more moderate in the sideline configuration. This can be attributed to the strong mean flow variations in the computational domain that are only partially captured by the interface conditions. Improved conditions should take into account the tangential components and derivatives of the mean flow.

Nevertheless, the second-order condition speeds up the approach configuration by a factor 4.1 and the sideline configuration by 1.9 in terms of iterations and computational time. It also improves the accuracy of the solution in terms of global residual. With a simple modification of the zeroth order condition, the second-order condition with the rotation branch-cut technique allows to physically act on the damping of evanescent modes. This results in a lower number of iterations by an amount that is comparable to the gain from an optimized second-order condition such as used in [16].

Finally we compare the benefit of the domain decomposition approach with the one currently used in the industry, that is the inversion of the matrix formed by the discretization of the entire domain. This matrix is given to a parallel 


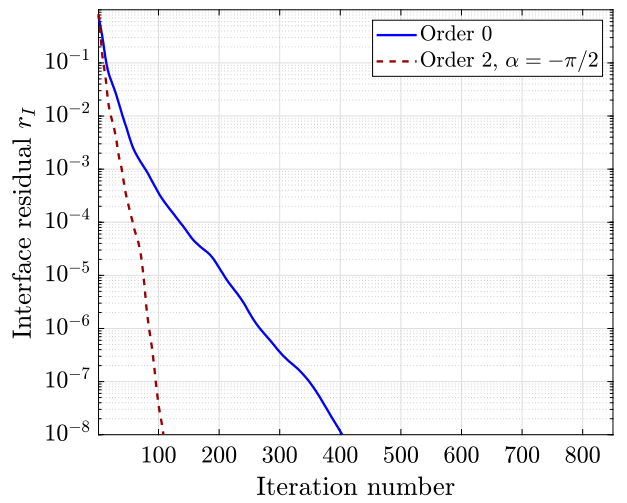

(a) Approach

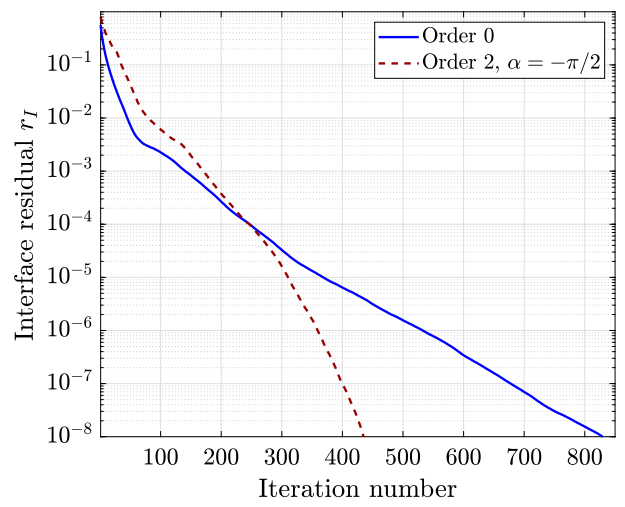

(b) Sideline

Fig. 7. Evolution of the interface residual for the interface conditions of order 0 and order 2 with rotation angle $\alpha=-\pi / 2$.

Table 4

Computational costs comparison between domain decomposition and standard methods for the sideline configuration. Each MPI process is assigned to a single-thread of an AMD Bulldozer $6272 \mathrm{CPU}$ at $2.1 \mathrm{GHz}$ with $256 \mathrm{~Gb}$ of RAM.

\begin{tabular}{lllll}
\hline & DDM - O2, $\alpha$ & DDM - O0 & MUMPS MPI & MUMPS \\
\hline Memory (LU + ORTHODIR) & $46 \mathrm{~Gb}+0.9 \mathrm{~Gb}$ & $46 \mathrm{~Gb}+0.9 \mathrm{~Gb}$ & $105 \mathrm{~Gb}$ & $440 \mathrm{~Gb}$ \\
Time (LU + ORTHODIR) & $1 \mathrm{~h} 52 \mathrm{~m}+2 \mathrm{~h} 6 \mathrm{~m}$ & $1 \mathrm{~h} 52 \mathrm{~m}+4 \mathrm{~h} 6 \mathrm{~m}$ & $22 \mathrm{~h} 40 \mathrm{~m}$ & - \\
\hline
\end{tabular}

version of MUMPS which splits the problem over 6 single-threaded MPI processes. We note that the domain decomposition problem globally contains a slightly higher number of degrees of freedom due to the creation of the interfaces unknowns. In the sideline configuration, the parallel version of MUMPS split on 6 processes requires 105 $\mathrm{Gb}$ of memory per node for the factorization. On a single process, the required memory increases to $440 \mathrm{~Gb}$. It is respectively 2.3 and 9.6 times more than the memory needed by the present domain decomposition technique for the factorization of the largest sub-domain matrix. With an appropriate transmission condition, the iterative solver then adds around $1 \mathrm{~Gb}$ of memory and an amount of computational time that is small compared to the factorization time of standard methods. The associated computational costs are summarized in Table 4. This confirms the high benefit of domain decomposition techniques for large-scale models and paves the way for the resolution of a new class of problems in flow acoustics.

\section{Conclusion}

A non-overlapping domain decomposition method, based on the optimized Schwarz approach, has been developed and verified for the simulation of sound with a background mean flow, namely the linearized potential equation propagation model. It was shown that, through the choice of suitable interface unknowns and conditions, it is possible to obtain a formulation that shares similar performance to the methods used for the Helmholtz problems. While adaptive high-order finite elements enhance the computational efficiency within the sub-domains, higher order interface conditions improve the performance and scalability of the global iterative procedure. With a simple physical parameter, the proposed second-order condition has shown to be more robust than the usual Robin-type condition and to perform as well as an equivalent optimized condition. On a real scale application, it results in a gain of a factor up to four in terms of iterations and computational time. Work is ongoing towards a better scalability of domain decomposition techniques with respect to the frequency, mean flow and sub-domains. The appropriate treatment of cross-points and the performance of optimized Schwarz in combination with the perfectly matched layers is also the topic of future research.

\section{Declaration of competing interest}

The authors declare that they have no known competing financial interests or personal relationships that could have appeared to influence the work reported in this paper. 


\section{Acknowledgments}

This work was performed as part of the CRANE project (Community and Ramp Aircraft NoisE, https://cordis.eur opa.eu/project/id/606844) funded by the European Union under the Seventh Framework Programme (Grant 606844) and under the CIFRE contract No 2018/1845 funded by Siemens Industry Software SAS and Association Nationale de la Recherche et de la Technologie (ANRT). This research was also funded in part through the ARC grant for Concerted Research Actions (ARC WAVES 15/19-03), financed by the Wallonia-Brussels Federation of Belgium. The authors acknowledge the use of the computational resources provided by the Consortium des Équipements de Calcul Intensif (CÉCI), funded by the Fonds de la Recherche Scientifique de Belgique (F.R.S.-FNRS) under Grant No. 2.5020.11 and by the Walloon Region. The authors would like to thank Prof. François-Xavier Roux for his helpful guidance on non-overlapping Schwarz domain decomposition methods.

\section{References}

[1] R. Astley, Numerical methods for noise propagation in moving flows, with application to turbofan engines, Acoust. Sci. Technol. 30 (4) (2009) 227-239.

[2] C. Farhat, F.-X. Roux, A method of finite element tearing and interconnecting and its parallel solution algorithm, Internat. J. Numer. Methods Engrg. 32 (6) (1991) 1205-1227.

[3] M. Gander, F. Magoules, F. Nataf, Optimized Schwarz methods without overlap for the Helmholtz equation, SIAM J. Sci. Comput. 24 (1) (2002) 38-60.

[4] Y. Boubendir, X. Antoine, C. Geuzaine, A quasi-optimal non-overlapping domain decomposition algorithm for the Helmholtz equation, J. Comput. Phys. 231 (2) (2012) 262-280.

[5] B. Thierry, A. Vion, S. Tournier, M. El Bouajaji, D. Colignon, N. Marsic, X. Antoine, C. Geuzaine, GetDDM: An open framework for testing optimized Schwarz methods for time-harmonic wave problems, Comput. Phys. Comm. 203 (2016) 309-330.

[6] V. Dolean, M.J. Gander, L. Gerardo-Giorda, Optimized Schwarz methods for Maxwell's equations, SIAM J. Sci. Comput. 31 (3) (2009) 2193-2213.

[7] M. El Bouajaji, B. Thierry, X. Antoine, C. Geuzaine, A quasi-optimal domain decomposition algorithm for the time-harmonic Maxwell's equations, J. Comput. Phys. 294 (2015) 38-57.

[8] V. Rawat, J.-F. Lee, Nonoverlapping domain decomposition with second order transmission condition for the time-harmonic Maxwell's equations, SIAM J. Sci. Comput. 32 (6) (2010) 3584-3603.

[9] C. Farhat, A. Macedo, M. Lesoinne, F.-X. Roux, F. Magoulés, A. de La Bourdonnaie, Two-level domain decomposition methods with Lagrange multipliers for the fast iterative solution of acoustic scattering problems, Comput. Methods Appl. Mech. Engrg. 184 (2-4) (2000) 213-239.

[10] B. Després, Décomposition de domaine et problème de Helmholtz, C. R. Math. Acad. Sci. Paris 1 (6) (1990) $313-316$.

[11] M. Myers, On the acoustic boundary condition in the presence of flow, J. Sound Vib. 71 (3) (1980) $429-434$.

[12] A. Bermúdez, L. Hervella-Nieto, A. Prieto, et al., An optimal perfectly matched layer with unbounded absorbing function for time-harmonic acoustic scattering problems, J. Comput. Phys. 223 (2) (2007) 469-488.

[13] A. Bermúdez, L. Hervella-Nieto, A. Prieto, R. Rodríguez, Perfectly matched layers, in: S. Marburg, B. Nolte (Eds.), Computational Acoustics of Noise Propagation in Fluids - Finite and Boundary Element Methods, Springer, 2008, pp. 167-196.

[14] V. Dolean, P. Jolivet, F. Nataf, An Introduction to Domain Decomposition Methods: Algorithms, Theory, and Parallel Implementation, Vol. 144, SIAM, 2015.

[15] P.-L. Lions, On the Schwarz alternating method. III: a variant for nonoverlapping subdomains, in: Third International Symposium on Domain Decomposition Methods for Partial Differential Equations, Vol. 6, SIAM Philadelphia, PA, 1990, pp. $202-223$.

[16] M. Gander, Optimized Schwarz methods, SIAM J. Numer. Anal. 44 (2) (2006) 699-731.

[17] X. Antoine, H. Barucq, A. Bendali, Bayliss-Turkel-like radiation conditions on surfaces of arbitrary shape, J. Math. Anal. Appl. 229 (1) (1999) 184-211.

[18] F.A. Milinazzo, C.A. Zala, G.H. Brooke, Rational square-root approximations for parabolic equation algorithms, J. Acoust. Soc. Am. 101 (2) (1997) 760-766.

[19] A. Modave, A. Royer, X. Antoine, C. Geuzaine, An optimized Schwarz domain decomposition method with cross-point treatment for time-harmonic acoustic scattering, 2020, Preprint HAL-02432422.

[20] Y. Saad, Iterative Methods for Sparse Linear Systems, Society for Industrial and Applied Mathematics, 2003.

[21] H. Bériot, A. Prinn, G. Gabard, Efficient implementation of high-order finite elements for Helmholtz problems, Internat. J. Numer. Methods Engrg. 106 (3) (2016) 213-240.

[22] G. Gabard, H. Bériot, A. Prinn, K. Kucukcoskun, Adaptive, high-order finite-element method for convected acoustics, AIAA J. 56 (8) (2018) 3179-3191

[23] P. Šolín, K. Segeth, I. Doležel, Higher-Order Finite Element Methods, Chapman \& Hall, New York, 2003.

[24] P.R. Amestoy, I.S. Duff, J. Koster, J.-Y. L'Excellent, A fully asynchronous multifrontal solver using distributed dynamic scheduling, SIAM J. Matrix Anal. Appl. 23 (1) (2001) 15-41.

[25] P.R. Amestoy, A. Guermouche, J.-Y. L'Excellent, S. Pralet, Hybrid scheduling for the parallel solution of linear systems, Parallel Comput. 32 (2) (2006) 136-156.

[26] A. Prinn, Efficient Finite Element Methods for Aircraft Engine Noise Prediction (Ph.D. thesis), University of Southampton, 2014 
[27] E. Bécache, A.-B. Dhia, G. Legendre, Perfectly matched layers for the convected Helmholtz equation, SIAM J. Numer. Anal. 42 (1) (2004) 409-433.

[28] P. Marchner, H. Beriot, X. Antoine, C. Geuzaine, Stable perfectly matched layers with Lorentz transformation for the convected Helmholtz equation, 2020, Preprint HAL-02556182.

[29] F. Nataf, A new approach to perfectly matched layers for the linearized Euler system, J. Comput. Phys. 214 (2) (2006) 757-772.

[30] G. Karypis, V. Kumar, A fast and highly quality multilevel scheme for partitioning irregular graphs, SIAM J. Sci. Comput. 20 (1) (1999) 359-392.

[31] H. Bériot, G. Gabard, E. Perrey-Debain, Analysis of high-order finite elements for convected wave propagation, Internat. J. Numer. Methods Engrg. 96 (11) (2013) 665-688.

[32] M. Bonazzoli, V. Dolean, I.G. Graham, E.A. Spence, P.-H. Tournier, Two-level preconditioners for the Helmholtz equation, in: International Conference on Domain Decomposition Methods, Springer, 2017, pp. 139-147.

[33] I. Graham, E. Spence, E. Vainikko, Domain decomposition preconditioning for high-frequency Helmholtz problems with absorption, Math. Comp. 86 (307) (2017) 2089-2127.

[34] A. Vion, C. Geuzaine, Double sweep preconditioner for optimized Schwarz methods applied to the Helmholtz problem, J. Comput. Phys. 266 (2014) 171-190.

[35] A. Vion, C. Geuzaine, Improved sweeping preconditioners for domain decomposition algorithms applied to time-harmonic Helmholtz and Maxwell problems, ESAIM Proc. Surv. 61 (2018) 93-111.

[36] M. Taus, L. Zepeda-Núñez, R.J. Hewett, L. Demanet, L-Sweeps: A scalable, parallel preconditioner for the high-frequency Helmholtz equation, 2019, arXiv preprint arXiv:1909.01467.

[37] M.J. Gander, H. Zhang, A class of iterative solvers for the Helmholtz equation: Factorizations, sweeping preconditioners, source transfer, single layer potentials, polarized traces, and optimized Schwarz methods, SIAM Rev. 61 (1) (2019) 3-76.

[38] P. Mustafi, Improved Turbofan Intake Liner Design and Optimization (Ph.D. thesis), University of Southampton, 2013.

[39] H. Bériot, G. Gabard, Anisotropic adaptivity of the p-FEM for time-harmonic acoustic wave propagation, J. Comput. Phys. 378 (2019) 234-256. 